\title{
Prevalencia de enterobiosis, su relación con la calidad de vida y calidad ambiental en la población de cinco instituciones educativas de inicial de Nuevo Tumbes durante 2010.
}

\section{Prevalence of enterobiasis, its relationship with the quality of life and environmental in the town of five initial educational institutions of Nuevo Tumbes during 2010}

\author{
Mirian Larrea*, Carlos Zamora**, Teresa Quevedo***, \\ Irene Urbina****
}

DOI: http://dx.doi.org/10.21503/CienciayDesarrollo.2012.v15i2.04

\section{RESUMEN}

Se determino la enterobiosis en niños de cinco Instituciones Educativos de Inicial; Carrusel de $\mathrm{Ni}$ ños, Mis Primero Pasos, Virgen del Cisne, Virgen de Fátima y Jorge Guimac Bonifaz, de Nuevo Tumbes "Puyango" y su relación con su calidad de vida y ambiental. El objetivo fue determinar la prevalencia de enterobiosis, y su relación con la calidad vida y ambiental de los estudiantes, que nos permita tomar acciones correctivas al problema. Se estudió una muestra de niños de ambos sexos, se hizo el estudio parasitológico, utilizando el Test de Graham, en cada unidad de estudio (alumno), se practicó el estado nutricional según técnica (IMC) de Quetelec; determinándose las condiciones de calidad ambiental (agua limpia potable, servicios higiénicos adecuados y energía eléctrica). La población fue de 596 alumnos. En relación a la calidad ambiental el $96,77 \%$ conto con energía eléctrica, 90,43\% con agua potable, $78,18 \%$ con un sistema de eliminación de excreta, $82,71 \%$ vivienda de construcción de materia noble y 65,82 $\%$ con domicilio en urbanización. Según el índice de masa corporal (IMC) delgadez aceptable 39,93\%, delgadez moderada $32.04 \%$, peso normal $16,94 \%$ delgadez severa $10,90 \%$ y sobre peso $0,33 \%$. La prevalencia de la enterobiosis en Mis Primeros Pasos 14,18\%, Carrusel de Niños 08,66\%. Jorge Guimac Bonifaz 04,43\%. Virgen del Cisne y Virgen de Fátima $02,85 \%$. Se concluye: La prevalencia de enterobiosis en la población de las cinco I.E.I. de Nuevo Tumbes fue positiva (07,55). El Estado nutricional: delgadez aceptable (39,93\%) delgadez moderada (32.04\%), peso normal (16,94\%), delgadez severa (10,90\%), y sobre peso $(0,33 \%)$.

Palabras claves: Enterobiosis, calidad de vida, calidad ambiental

\section{ABSTRACT}

It was determined the enterobiasis in five kindergarten institutions: "Carrusel de Niños, Mis Primeros Pasos, Virgen del Cisne, Virgen de Fátima and Jorge Guimac Bonifaz" of Nuevo Tumbes "Puyango" and its relation with the enviromental and life quality of the students. The objective was determining the prevalence of enterobiasis and its relation with the environmental and life quality of the students that

\footnotetext{
*Biologo microbiologo, Doctor en salud publica Universidad Nacional de Trujillo, Facultad de Ciencias de la Salud. E-mail: larreamirian@yahoo.com

${ }^{* *}$ Biologo Pesquero, Doctor en Ciencias Biomedicas, Universidad Alas Peruanas - Filial Tumbes. E-mail: kalinzamg@gmail.com

${ }^{* * *}$ Quimico Farmaceutico, Doctora en Ciencias De La Salud, Universidad Nacional De Tumbes-Facultad De Ciencias De La Salud.

E-mail: teresaquevedo@gmail.com

${ }^{* * * *}$ Licenciada en enfermeria, doctora en ciencias de enfermeria, universidad nacional de tumbes-facultad de ciencias de la salud. E-mail: yeur2020@hotmail.Com
} 
allow us to take corrective actions to the problem. It was studied a sample of boys of both sex. It was done the parasitological study, using the Graham test; in each study unit (student), it was practiced the nutritional state according (BMI) of Quetelec; determining the environmental conditions (tap water, suitable toilets, and electric energy). The population was 596 students. In relation with the environmental quality, the $96.77 \%$ got electric energy, $90.43 \%$ with drinking water, $78.18 \%$ with a excreta elimination system, the $82.71 \%$ with a masonry house and $65.82 \%$ living in urbanizations. According the body mass (BMI) an acceptable thinness is $39.93 \%$, moderate thinness is $32.04 \%$, normal weight $16.94 \%$, severe thinness $10.90 \%$ and overweight is $0.33 \%$. Enterobiasis prevalence in "Mis Primeros Pasos" was $14.18 \%$, "Carusel de niños" 08.66\%, "Jorge Guimac Bonifaz" 04.43\%, "Virgen del Cisne" and "Virgen de Fàtima" $02.85 \%$, We conclude: the enterobiasis prevalence in population of the five kindergarten in Nuevo Tumbes was positive (07.55). The nutritional state was: acceptable thinness $(39.93 \%)$, moderate thinness (32.04\%), normal weight (16.94\%), severe thinness (10.90\%) and overweight $(0.33 \%)$.

Keywords: Enterobiasis, life quality, environmental quality.

\section{INTRODUCCIÓN}

Los niños son sin duda los más vulnerables frente al riesgo de adquirir estas infecciones y sus efectos, debido a su inmadurez inmunológica. Una vez que un niño es infectado, la probabilidad de contagiar a sus familiares cercanos es alta, por su escasa práctica de buenos hábitos higiénicos (Iannacone et al., 2006). Para que el problema persista o se agudice, es necesario que los gobiernos organicen el acceso al tratamiento quimioterápico periódico, aspecto que la Organización Mundial de la Salud se halla incentivando a través de sus Proyectos de Salud Escolar (WHO, 2010).

Actualmente se evidencia que diferentes grupos poblacionales de niños presentan condiciones de riesgo que facilitan la infestación por enteroparasitosis, la misma que provoca impactos negativos en la calidad de vida de los niños. En la población infantil que padece de notorias deficiencias en su dieta alimentaria, los parásitos encuentran un terreno apto para vivir y prosperar contribuyendo a agravar aún más su desnutrición.

En grado mayor o menor los parásitos intestinales privan a su hospedero humano de ciertos nutrientes, y por lo tanto favorecen la anemia, la desnutrición y la susceptibilidad a otras enfermedades, desfavoreciendo el proceso del apren- dizaje (Botero y Restrepo, 1997). La presente investigación tuvo como objetivo identificar la frecuencia de enteroparasitosis y su relación con la calidad vida y ambiental en niños de cinco instituciones educativos de inicial: Carrusel de $\mathrm{Ni}$ ños, Mis Primeros Pasos, Virgen del Cisne, Virgen de Fátima y Jorge Guimac Bonifaz, ubicados en Nuevo Tumbes, "Puyango".

\section{MATERIAL Y MÉTODOS}

La muestra estuvo constituida por los 596 niños que acudieron a los centros educativos primarios de Nuevo Tumbes y que reunieron los criterios de inclusión. El criterio de inclusión fue que todos los niños de 3 a 5 años de edad asistieran a las instituciones educativas de inicial y que proporcionaran la muestra biológica. Para la obtención de las muestras fecales, a cada niño/padre de familia se le realizó una encuesta socioeconómica, y se le entregó un portaobjetos estéril, dándole las indicaciones del caso para la obtención de la muestra (Test de Graham), haciendo hincapié en las medidas de higiene.

En lo que toca a la calidad de vida y ambiental, se consideró en el test socioeconómico las condiciones urbanísticas y ambientales, los servicios con que cuentan, como son agua potable, desagüe y fluido eléctrico, y finalmente el tipo de construcción de las viviendas. 
La obtención del índice de masa corporal se realizó tomando a los alumnos el peso y la talla. El índice de masa corporal (IMC) se determinó según la técnica de Quetelec.

Para el análisis estadístico, las variables se procesaron en una hoja de cálculo Excel-Windows, y en los análisis correspondientes se caracterizaron a los grupos positivos y negativos de acuerdo a las variables analizadas, obteniéndose las tablas respectivas. Los resultados fueron tabulados relacionando los casos positivos, y teniendo en cuenta los parámetros de edad y sexo. En relación con la calidad de vida, se consideró el tipo de material empleado en la construcción de su domicilio.

\section{RESULTADOS}

Tabla 1: La calidad ambiental para los alumnos de las cinco Instituciones Educativas de Inicial de Nuevo Tumbes: Carrusel de Niños, Mis Primeros Pasos, Virgen del Cisne, Virgen de Fátima y Jorge Guimac Bonifaz. Durante el 2010. Tumbes Perú.

\begin{tabular}{|c|c|c|c|c|c|c|c|c|c|c|c|c|c|}
\hline \multirow{8}{*}{ 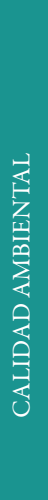 } & \multirow{2}{*}{$\begin{array}{l}\text { INSTITUCIONES } \\
\text { EDUCATIVA DE INICIAL }\end{array}$} & \multicolumn{2}{|c|}{$\begin{array}{l}\text { CARRUSEL DE } \\
\text { NIÑOS }\end{array}$} & \multicolumn{2}{|c|}{$\begin{array}{l}\text { MIS PRIMEROS } \\
\text { PASOS }\end{array}$} & \multicolumn{2}{|c|}{$\begin{array}{l}\text { VIRGEN DEL } \\
\text { CISNE }\end{array}$} & \multicolumn{2}{|c|}{$\begin{array}{l}\text { VIRGEN DE } \\
\text { FÁTIMA }\end{array}$} & \multirow{2}{*}{\multicolumn{2}{|c|}{$\begin{array}{l}\text { JORGE } \\
\text { GUIMAC } \\
\text { BONIFAZ }\end{array}$}} & \multicolumn{2}{|c|}{$\begin{array}{l}\text { POBLACIÓN } \\
\text { TOTAL }\end{array}$} \\
\hline & & $\mathrm{N}$ & $\%$ & $\mathrm{~N}$ & $\%$ & $\mathrm{~N}$ & $\%$ & $\mathrm{~N}$ & $\%$ & & & $\mathrm{~N}$ & $\%$ \\
\hline & AGUA POTABLE & 144 & 96.00 & 135 & 91,21 & 50 & 71,42 & 60 & 85.71 & 150 & 94,93 & 539 & 90,43 \\
\hline & CORRIENTE ELÉCTRICA & 150 & 100 & 138 & 93,24 & 59 & 84,28 & 63 & 90,00 & 137 & 86,70 & 547 & 91,77 \\
\hline & $\begin{array}{l}\text { SISTEMA DE ELIMINACIÓN DE } \\
\text { EXCRETAS }\end{array}$ & 142 & 94.66 & 142 & 95,94 & 46 & 65,71 & 61 & 87,14 & 75 & 47,46 & 466 & 78,18 \\
\hline & $\begin{array}{l}\text { UBICACIÓN DE LA URBANIZACIÓN } \\
\text { VIVIENDA }\end{array}$ & 135 & 90.00 & 145 & 97,97 & 28 & 40,00 & 67 & 95,71 & 107 & 67,72 & 482 & 80,87 \\
\hline & $\begin{array}{c}\text { MATERIAL } \\
\text { CONSTRUCCIÓN }\end{array}$ & 132 & 88.00 & 140 & 94,59 & 50 & 71,42 & 67 & 95,71 & 104 & 65,82 & 493 & 82,71 \\
\hline & POBLACIÓN & 150 & 25,16 & 148 & 24,86 & 70 & 11,75 & 70 & 11,75 & 158 & 26,56 & 596 & 100 \\
\hline
\end{tabular}

Tabla 2: El estado nutricional para los alumnos de las cinco I.E.I. de Nuevo Tumbes : Carrusel de Niños, Mis Primeros Pasos, Virgen del Cisne, Virgen de Fátima y Jorge Guimac Bonifaz. Durante el 2010. Tumbes Perú.

\begin{tabular}{|c|c|c|c|c|c|c|c|c|c|c|c|c|c|}
\hline \multirow{2}{*}{\multicolumn{2}{|c|}{$\begin{array}{c}\text { INSTITUCIONES } \\
\text { EDUCATIVA DE } \\
\text { INICIAL }\end{array}$}} & \multicolumn{2}{|c|}{$\begin{array}{c}\text { CARRUSEL DE } \\
\text { NIÑOS }\end{array}$} & \multicolumn{2}{|c|}{$\begin{array}{c}\text { MIS PRIMEROS } \\
\text { PASOS }\end{array}$} & \multicolumn{2}{|c|}{$\begin{array}{l}\text { VIRGEN DEL } \\
\text { CISNE }\end{array}$} & \multicolumn{2}{|c|}{$\begin{array}{l}\text { VIRGEN DE } \\
\text { FÁTIMA }\end{array}$} & \multicolumn{2}{|c|}{$\begin{array}{c}\text { JORGE GUIMAC } \\
\text { BONIFAZ }\end{array}$} & \multirow{2}{*}{\multicolumn{2}{|c|}{$\begin{array}{c}\text { POBLACION } \\
\text { TOTAL }\end{array}$}} \\
\hline & & $\mathrm{N}$ & $\%$ & $\mathrm{~N}$ & $\%$ & $\mathrm{~N}$ & $\%$ & $\mathrm{~N}$ & $\%$ & $\mathrm{~N}$ & $\%$ & & \\
\hline & $\begin{array}{l}\text { DELGADEZ } \\
\text { SEVERA }\end{array}$ & 35 & 23,33 & 30 & 27,70 & 00 & 00 & 00 & 00 & 00 & 00 & 65 & 10,90 \\
\hline 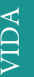 & $\begin{array}{l}\text { DELGADEZ } \\
\text { MODERADA }\end{array}$ & 49 & 32,66 & 31 & 20,94 & 06 & 08,57 & 27 & 38,57 & 78 & 49,36 & 191 & 32,04 \\
\hline$\frac{1}{0}$ & $\begin{array}{l}\text { DELGADEZ } \\
\text { ACEPTABLE }\end{array}$ & 45 & 30,00 & 41 & 27,70 & 58 & 82,85 & 28 & 40,00 & 66 & 41,77 & 238 & 39,93 \\
\hline 峞 & $\begin{array}{l}\text { PESO } \\
\text { NORMAL }\end{array}$ & 21 & 14,00 & 46 & 31,08 & 05 & 07,14 & 15 & 21,42 & 14 & 08,86 & 101 & 16,94 \\
\hline 0 & SOBRE PESO & 00 & 00 & 00 & 00 & 02 & 02.85 & 00 & 00 & 00 & 00 & 02 & 0,33 \\
\hline & POBLACIÓN & 150 & 25,16 & 148 & 24,83 & 70 & 11,75 & 70 & 11,75 & 158 & 26,56 & 596 & 100 \\
\hline
\end{tabular}


Tabla 3: Prevalencia de enterobiosis en relación al sexo y la edad en los alumnos de las cinco I. E. I. de Nuevo Tumbes: Carrusel de Niños, Mis Primeros Pasos, Virgen del Cisne, Virgen de Fátima y Jorge Guimac Bonifaz. Durante el 2010. Tumbes Perú.

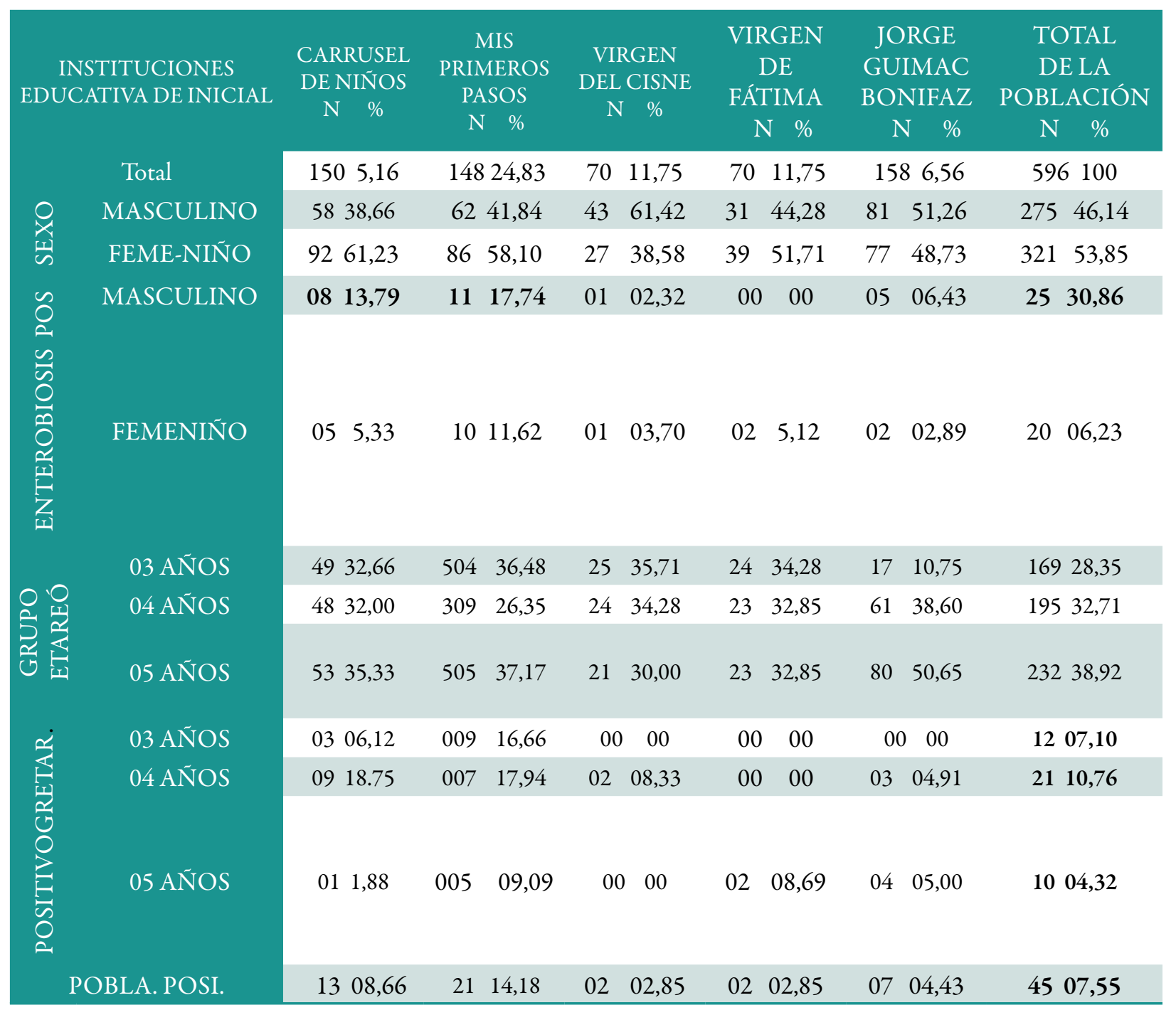


Tabla 4: Se muestra la relación entre, la calidad de vida, la calidad ambiental y la prevalencia de la enterobiosis, de los alumnos de las cinco I. E.I de Nuevo Tumbes : Carrusel de niños, Mis primeros Pasos, Virgen de Fátima, Virgen del Cisne y Jorge Guimac Bonifaz. Durante el 2010. Tumbes Perú.

\begin{tabular}{|c|c|c|c|c|c|c|c|c|}
\hline \multicolumn{3}{|c|}{ INSTITUCIONES EDUCATIVA DE INICIAL } & $\begin{array}{c}\text { CARRUSEL } \\
\text { DE NIÑOS } \\
\%\end{array}$ & $\begin{array}{c}\text { MIS } \\
\text { PRIMEROS } \\
\text { PASOS } \\
\%\end{array}$ & $\begin{array}{c}\text { VIRGEN } \\
\text { DEL CISNE } \\
\%\end{array}$ & $\begin{array}{c}\text { VIRGEN DE } \\
\text { FÁTIMA } \\
\%\end{array}$ & $\begin{array}{c}\text { JORGE } \\
\text { GUIMAC } \\
\text { BONIFAZ } \\
\%\end{array}$ & $\begin{array}{c}\text { TOTAL } \\
\text { DE LA } \\
\text { POBLACIÓN } \\
\%\end{array}$ \\
\hline \multirow{5}{*}{ 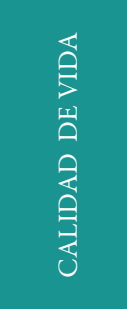 } & & $\begin{array}{l}\text { GADEZ } \\
\text { EVERA }\end{array}$ & 23,33 & 20,70 & 00 & 00 & 00 & 10,90 \\
\hline & $\begin{array}{l}\mathrm{DE} \\
\mathrm{MC}\end{array}$ & $\begin{array}{l}\text { GADEZ. } \\
\text { DERADA }\end{array}$ & 32,66 & 20,94 & 08,57 & 38,57 & 49,36 & 32,04 \\
\hline & & $\begin{array}{l}\text { GADEZ } \\
\text { PTABLE }\end{array}$ & 30,60 & 27,70 & 12,15 & 40,00 & 41,77 & 39,93 \\
\hline & PESC & NORMAL & 14,00 & 31,08 & 07,14 & 21,42 & 08,86 & 16,94 \\
\hline & $\mathrm{SOl}$ & RE PESO & 00 & 00 & 02,85 & 00 & 00 & 0,33 \\
\hline \multirow{7}{*}{ 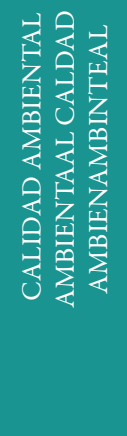 } & AGU & POTABLE & 96,00 & 91,21 & 71,42 & 85,71 & 94,93 & 90,43 \\
\hline & ENERGI & ELECTRICA & 100 & 93,24 & 59,28 & 90,00 & 86,70 & 96,77 \\
\hline & $\begin{array}{c}S \\
\text { EX }\end{array}$ & $\begin{array}{l}\text { ST. ELI. } \\
\text { CRETA }\end{array}$ & 94,66 & 95,94 & 65,71 & 87,14 & 47,46 & 78,18 \\
\hline & $\begin{array}{l}\text { UBICA. } \\
\text { VIVIENDA }\end{array}$ & URBANIZACIÓN & 90,00 & 97,97 & 40,00 & 95,97 & 67,72 & 65,82 \\
\hline & $\begin{array}{l}\text { MATERIAL } \\
\text { DE CASA }\end{array}$ & $\begin{array}{c}\text { MATERIAL } \\
\text { NOBLE }\end{array}$ & 88,00 & 94,59 & 71,52 & 95,71 & 65,82 & 82,71 \\
\hline & ENTEROBI & OSIS & 08,66 & 14,18 & 02,85 & 02,85 & 04,43 & 07,55 \\
\hline & TOTAI & & 25,16 & 24,83 & 11,75 & 11,75 & 26,56 & 100 \\
\hline
\end{tabular}

\section{DISCUSIÓN}

En la presente investigación se cumplió con las normas éticas y se eligieron las relaciones entre las condiciones ambientales, la calidad de vida, la prevalencia de la enterobiosis, y a su vez se consideró el género, porque aún hay controversia acerca de si el parasitismo intestinal se relaciona con el sexo, También se tomó en cuenta el lugar donde está ubicada la vivienda, aspecto directamente relacionado con el parasitismo, ya que este fenómeno es mayor en zonas rurales porque carecen de saneamiento básico (Iannacone et al., 2006). El otro grupo de factores correspondió a los ambientales, y comprendió la disponibilidad de agua para beber, porque muchos parásitos, sobre todo los protozoarios, tienden a ingresar al organismo con este elemento (Ekpo et al., 2008), y el lugar de defecación, porque los parásitos por su ubicación salen con la materia fecal y contaminan el ambiente, de donde regresan por varios mecanismos al organismo de los escolares (Putignami y Menichella, 2010).

De la población estudiantil de las instituciones educativas de inicial de Nuevo Tumbes, el 91,43 \% contó con energía eléctrica, el 90,43\% con agua potable, el $78,18 \%$ con un sistema de eliminación de excretas; el 80,87 \% de las viviendas se ubicó en zona de urbanización, y el 82,71\% estaban construidas de material noble. En relación con las instituciones educativas de inicial, presentaron en un $100 \%$ energía eléctrica, y el $96,00 \%$ tenía agua potable. Se determinó que de los escolares de la I.E.I. Carrusel de Niños, el 97,97 \% viven en urbanizaciones; la I.E.I. Mis Primeros Pasos presentó en un 95,94\% un sistema de eliminación de excretas. En la I.E.I. Virgen de Fátima se encontró que el 95,71 \% había utilizado en la construcción de su vivienda material noble. Las condiciones ambientales más bajas se presentaron en la población de la I.E.I. Virgen 
del Cisne, y la mejor calidad ambiental la presentó Carrusel de Niños (Tabla 1). Los resultados en cuanto a los factores ambientales estudiados demostraron que las condiciones son mejores que los reportadas para toda la población peruana por INEI-ENAHO (1999), en donde, a nivel nacional, el 60,4\% de las viviendas de zonas rurales son de paredes de estera o piso de tierra, el 64,2\% de viviendas presentan red de agua pública, el 50,8\% de viviendas presentan desagües conectados a red pública, el 4,4\% se abastece de agua por camión cisterna de reparto, el 6,9 $\%$ de pozo y el $17,9 \%$ de un río, acequia o un manantial. En lo que se refiere al abastecimiento por cisterna y pozo encontramos un $6,2 \%$ y $8,6 \%$, respectivamente, que en este aspecto no ha mejorado, a pesar de existir proyectos nacionales de programa de agua y saneamiento en la región fronteriza, como el establecido por la Ley 29142 a través del Programa Agua para Todos. Lo cierto es que el crecimiento poblacional en los AA.HH. es mayor a la elaboración, aprobación y ejecución de las políticas de gobierno.

Zamora (2009) demuestra la prevalencia del enteroparasitismo $(90,6 \%)$ en la población escolar de Nuevo Tumbes (Tumbes, Perú), en relación con factores sociodemográficos, ambientales y con el rendimiento académico; encontrando que la población escolar estuvo compuesta en un porcentaje ligeramente mayor por escolares del sexo masculino que femenino (53,2 vs. 47,8), y que el mayor volumen de escolares (85,2\%) vivía en los denominados asentamientos humanos. Asimismo, que la mayoría $(50,4 \%)$ de viviendas estuvieron confeccionadas con material de la región $(84$ $\%)$, tenían agua entubada y baño intradomiciliar $74,1 \%$, la gran mayoría $(86,1 \%)$ vivían en asentamientos humanos, y cuando se relacionaron las prevalencias con los factores ambientales, se encontró que los escolares de las I.E.P. que habitaban en viviendas construidas con materiales de la región (53,0 \%) estuvieron significativa- mente más parasitados; dichas viviendas tenían agua potable $(85,20 \%)$ e inodoro intradomiciliar $(77,0 \%)$, valores significativamente mayores $(\mathrm{p}<0,05)$ que sus variables contrincantes: agua de pozo o pozo ciego u otro sistema, respectivamente. Se encontró que el parasitismo intestinal afecta el rendimiento escolar, lo que no se acerca a los resultados obtenidos en la población de las cinco I.E.I., porque los que tienen una calidad ambiental mínima no presentan la más altas prevalencias, que en este caso son Virgen del Cisne y Jorge Guimac Bonifaz.

Según el índice de masa corporal (IMC) en la población escolar de las cinco I.E.I., en relación con cada una de las I.E.I. (Tabla 2): Virgen del Cisne presentó delgadez aceptable (41,77\%), Jorge Guimac Bonifaz presentó delgadez moderada (49,36 \%), Mis Primeros Pasos presentó peso normal (31,08\%), Carrusel de Niños presentó delgadez severa $(23,33 \%)$ y Virgen del Cisne presentó sobrepeso $(02,85 \%)$. Para determinar la relación entre la calidad de vida y la prevalencia de la enterobiosis en la población, se debe tener en cuenta la delgadez moderada, que fue la que se presentó con el valor más alto (49,36\%), y la prevalencia de la enterobiosis fue del 07,55 \% en la población, presentando esta última los valores más altos en relación con la primera. Si hubiera relación, los valores obtenidos estarían cercanos o iguales, lo que no ha ocurrido en esta población escolar, por lo que se puede afirmar que no hay relación entre calidad de vida y la prevalencia de la enterobiosis en las cinco I.E.I. de Nuevo Tumbes.

La prevalencia de la enterobiosis en los escolares de las cinco I.E.I. de Nuevo Tumbes fue de 07,55 $\%$. La prevalencia más alta en relación con el sexo la presentó la población masculina $(30,86$ $\%)$, y en relación con la edad se presentó en niños de 5 años con 38,92\%, y se encontró que los niños se hallaban más parasitados que las niñas (Tabla 3), lo que indica que los niños estarían 
más expuestos a estas enteroparasitosis, y por lo tanto presentan un alto riesgo de infección por parásitos; sin embargo, tanto niños como niñas tienen las mismas posibilidades de infectarse con las mismas consecuencias, debido a que la conformación del aparato digestivo y el funcionamiento del mismo no tiene diferencias respecto al sexo, puesto que los hábitos alimenticios son similares en ambos y también las oportunidades de infección por parásitos. Ibáñez et al. (2004) y Zamora (2009) coinciden en cuanto a la relación de infestación con el sexo; y cuando se relacionaron las prevalencias con los factores sociodemográficos, se encontró que un ligero porcentaje mayor de varones sufría infecciones respecto de las mujeres ( 59,7 vs. 40,3$)$.

En relación con los factores ambientales, se determinó que sí tiene efecto sobre la prevalencia de la enterobiosis; sin embargo, se debe considerar que los niños pasan la mayor parte del tiempo en las instituciones educativas, donde también consumen alimentos: los que llevan en la lonchera o los que adquieren de personas que las venden como comida ambulatoria, la misma que comparten o intercambian (Zamora, 2009), y lo hacen sin las normas de bioseguridad mínimas, por el desconocimiento de las mismas, como es el lavado de manos. Esta es precisamente la forma de la diseminación de los huevos de la enterobiosis, que llegan a las manos por el rascado o por el prurito anal en los niños que presentan este tipo de infestación. Esto hace que dejen la forma infectante (huevos) en todo lo que está en contacto con sus manos.

Si se relaciona la prevalencia de la enterobiosis con la calidad de vida de las poblaciones, el índice de masa corporal (IMC) en la población escolar de las cinco I.E.I. nos indica que la delgadez aceptable se presentó en 39,93 \%, la delgadez moderada en $32,04 \%$, el peso normal en 16,94 $\%$, la delgadez severa en el $10,90 \%$, y el sobrepeso en $0,33 \%$. La I.E.I. que presentó una alta pre- valencia de infestación por enterobiosis fue Mis Primeros Pasos, con 14,18 \%. Si la calidad de vida y la frecuencia de la enterobiosis tuvieran relación, esta se debió presentar en la población con delgadez severa, es decir, en Carrusel de niños, con el 23,33\%. Asimismo, una alta prevalencia a la enterobiosis debería presentarse en Mis Primeros Pasos, por presentar alta desnutrición, lo que no concuerda con los resultados hallados (Tabla 4). No hay, pues, relación inversa entre calidad de vida y ambiental y la prevalencia de la enterobiosis, lo que llevaría a pensar que el alto porcentaje de desnutrición es producto de la falta de educación y seguridad alimentaria, pero también del muy escaso presupuesto estatal y familiar destinado a la salud, reportado por UNICEF (2004).

\section{CONCLUSIONES}

La prevalencia de la enterobiosis en la población de las cinco instituciones educativas de inicial de Nuevo Tumbes fue positiva $(07,55 \%)$.

La prevalencia de la enterobiosis en las instituciones educativas de inicial se presentó según la siguiente relación: I.E.I. Mis Primeros Pasos 14,18 \%, I.E.I. Carrusel de Niños 08,66 \%, I.E.I. Jorge Guimac Bonifaz 04,43 \%.

La I.E.I. Jorge Guimac Bonifaz fue la que presentó delgadez moderada (49,38 \%) y delgadez aceptable (41,77\%), Mis Primeros Pasos presentó peso normal $(31,08 \%)$, Carrusel de Niños mostró delgadez severa $(23,33 \%)$, y Virgen del Cisne evidenció sobrepeso en $0,33 \%$.

Las condiciones ambientales más bajas las vivieron los escolares de la I.E.I. Virgen del Cisne, y la mejor calidad ambiental la presentó Carrusel de Niños. La prevalencia de enterobiosis no tiene relación con las condiciones de calidad de vida ni con las condiciones ambientales. 


\section{REFERENCIAS BIBLIOGRÁFICAS}

1. Botero y Restrepo. 2005. Parasitología bumana. Cuarta edición. Colombia: Edit. Corporación para las Investigaciones Biológicas.

2. Ekpo, E.; Odoemene, S.; Mafiana, C. y S. Sam-Wobo. 2008. "Helminthiasis and Hygiene Conditions of schools in Ikenne, OgunStatew, Nigeria”. PlosNeglectedTropDis (1): e 146.

3. Ibáñez, S. 2004. Manual de pediatria: desnutrición infantil. Disponible: [http.// wwwescuelamed/.puc.cl/paginas/publicaciones/ ManualPed/Desnu tric.htm] 20 de junio 2010.

4. Iannacone, J., Benites, M. y L. Chirinos. 2006. "Prevalencia de infección por parásitos intestinales en escolares de primaria de Santiago de Surco, Lima, Perú". ParasitolLatinoam, Lima, Perú.

5. INEI-ENAHO. 1999. Características de las viviendas y de los servicios básicos. Disponible en: [http.// www INEI.GOB.PE/BIBLIO INEIPUB /BANCOPUB /EST/LIB0078 /SO2-3HTM]. 20 de agosto de 2010.

6. Putignami, L. y D. Menichella. 2010. "Global distribution, public health and clinical impact of the protozoa pathogen Cryptosporidium”. Intersc Perspect Inf Dis. ID753512.

7. UNICEF. 2004. Por una niñez bien nutrida: comunicación para la acción. Disponible en: [http.// www unicef.org.co/pdf/ nutril_ pg1-9.pdf]. 16 de diciembre de 2009.

8. WORLD HEALTH ORGANIZATION WHO-. 2010. El medio ambiente y la salud de los niños. Healthy Environments for Children. Initiating an Aliance for Action. Doi: [www.who.int/social_determimnanants/ en/.fabainforma.htm]. 20 de enero de 2010, Perú.
9. Zamora, C. 2009. Prevalencia del enteroparasitismo en la población escolar de Nuevo Tumbes (Tumbes, Perú), su relación con factores sociodemográficos, ambientales y con el rendimiento académico. Tesis Doctoral en Biomédicas. Trujillo, Perú: Escuela de Postgrado, Universidad Nacional de Trujillo. 